Tequio 1(1), 2017: 32-38

ISSN: 2594-0546

\title{
CIUDADANÍA Y CULTURA CÍVICA EN ESTUDIANTES UNIVERSITARIOS INTERCULTURALES
}

\section{Graciela González-Juárez ${ }^{1}$}

Fecha de recepción: 4 de noviembre de 2016

Fecha de aceptación: 25 de mayo de 2017

Resumen - El objetivo de la investigación fue analizar la cultura cívica de los alumnos de universidades interculturales. Se examinó la actitud mostrada en situaciones emblemáticas, a fin de valorar formas de conducción respetuosa y armoniosa en espacios comunes de convivencia entre universitarios. El marco de referencia fue la sociología crítica para abordar los temas de ciudadanía y cultura cívica. La investigación fue descriptiva, con la participación total de 228 estudiantes de escuelas interculturales de tres regiones del país. Se reportan variaciones de acuerdo con el nivel del involucramiento percibido por los participantes en la situación planteada; en la medida en que se percibieron directamente involucrados en la situación hipotética, su actitud se alejó de la conducta más cívica, e incluso manifestaron estar dispuestos a incurrir en faltas para salir librados de ella. La cultura cívica se ve influida por variables históricas, sociales y culturales de la subjetividad de los sujetos.

Palabras clave:

Ciudadanía, cultura cívica, universitarios, interculturalidad, dilemas.
Abstract - The objective of the research was to analyze the civic culture of students in intercultural universities, through their attitude toward situations emblematic to assess ways of driving respectful and harmonious in common spaces of coexistence among academics. The frame of reference was critical sociology to address issues citizenship and civic culture. The research was descriptive and quantitative. A citizenship questionnaire was designed and applied to a total of 228 students in intercultural universities from three regions of the country. The results were processed in SPSS and obtained descriptive statistics for each considered situation. The findings show variations in each condition according to the level of involvement perceived by participants in the situation. As the participant was perceived to be directly involved in the hypothetical situation, its civic attitude moved away from more civic conduct and even expressed willingness to incur fouls to get rid of them. In the situation in which the participant is perceived less involved or disrupted by the dilemma posed, civic responses had the highest frequencies and there was less variability in responses. It is concluded that civic culture is influenced by historical, social and cultural variables that make up the subjectivity of the subjects, and deserve to be studied.

\section{Keywords:}

Citizenship, civic culture, academics, interculturality. 


\section{Ciudadanía y cultura cívica}

a ciudadanía es un concepto polisémico que se ha transformado de época en época. Ha estado presente desde la antigua polis griega, hasta las democracias modernas. Se entiende como "el conocimiento, la valoración positiva, la capacidad y la voluntad de los ciudadanos para disfrutar los derechos y cumplir las obligaciones que supone la condición ciudadana" (García Clarck, 2001, p. 446).

La noción de ciudadanía se articuló a la clase social con Thomas Marshall (1950), es un estatus que atribuye derechos y deberes a los nuevos grupos sociales en la relación Estado-sociedad e involucra tres dimensiones: civil, política y social. La ciudadanía supone la aceptación de los otros y las otras, incluyendo su cultura, valores, comportamientos y actitudes cívicas que les permiten relacionarse, interactuar y establecer vínculos interculturales y multiculturales. En la actualidad, las sociedades democráticas enaltecen la ciudadanía y el hecho de que individuos o grupos con culturas y razas de muy diverso origen puedan convivir y constituirse para funcionar en una sociedad plural.

En este sentido, la definición de ciudadanía tiene una articulación directa con la visión de Estado establecida en la Constitución y determina tanto su contenido como sus límites. Uno de sus componentes es la cultura cívica que, a su vez, tiene tres dimensiones a considerar: la primera es jurídicopolítica, que contempla los derechos y obligaciones de los individuos relativos a la participación en la vida pública; la segunda es la moral, referente a la aceptación voluntaria, aprecio y cultivo de los valores relacionados con la convivencia colectiva; la tercera, histórico-social, remite al sentido de pertenencia a una sociedad o nación constituida en Estado, a partir de un origen o rasgos comunes que identifican a sus integrantes (García Clarck, 2001). Para este autor, la cultura cívica y la ciudadanía son nociones equivalentes de distinta extensión política:
La cultura cívica o ciudadana, al igual que otros conceptos cercanos como cultura republicana o cultura democrática, remite al campo de la subjetividad política necesaria para la constitución y adecuado funcionamiento de los sistemas políticos. La diferencia entre los tres conceptos estriba, fundamentalmente, en su extensión, ya que el primero se puede aplicar a todo sistema político que requiera un cierto grado de participación de los ciudadanos para operar legítimamente. El segundo, en cambio, limita su aplicación a los sistemas republicanos y el tercero a los de carácter democrático (García Clarck, 2001, p. 446).

Desde la perspectiva de Almond y Verba (1970), en su estudio clásico La cultura cívica, ésta le corresponde a la democracia como cultura mixta, ya que combina tanto rasgos tradicionales como modernos. En los primeros, la cultura cívica de la democracia autoritaria implica que los individuos conserven el rol pasivo de súbditos, además de que les demanda obediencia y lealtad a la autoridad; el rol parroquial, por su parte, mantiene la vinculación predominante conel grupo primario. Adiferencia delos dos anteriores, la democracia moderna es más participativa e incorpora la función de participante abierto, racionalistaactivista del ciudadano (García Clarck, 2001).

La cultura cívica combina estos roles, así como los modelos de vinculación cerrada y abierta. Si bien es cierto que "el ciudadano informado, comprometido, racional y activo se encuentra con más frecuencia en las democracias que tienen éxito que en aquellas que no lo tienen, también lo es que la democracia sólo requiere parcialmente de este tipo de ciudadano" (Almond y Verba, 1970, p. 530).

De acuerdo con estos autores, el futuro de la democracia requeriría de la incorporación y adopción de sus principios en el funcionamiento de las instituciones y de la cultura cívica; esta última es una subcultura integrada por sistemas de creencias o códigos de relaciones personales cuyas propiedades son más difusas que las de una ideología o conjunto de normas legales, pero su influencia sobre el comportamiento 
de los actores políticos podría ser más decisiva. Así, se reconoce la importancia de las actitudes y sentimientos como insumos esenciales de la cultura cívica, así como la dificultad que requiere su aprendizaje. La asimetría parece una condición necesaria de las democracias modernas y de la cultura cívica de los ciudadanos. Cabe destacar el antagonismo de la democracia y la ciudadanía, ya que al tiempo que es deseable la presencia de ciudadanos activos, esto pondría en inestabilidad y crisis al sistema por sobredemanda ciudadana.

Así, mediante sus instituciones, la sociedad democrática aspira a capacitar al conjunto de sus ciudadanos para que tengan virtud cívica; es decir, que sean potencialmente capaces de tomar parte en la vida pública, al margen del grado en que decidan hacerlo, y en este sentido la educación universitaria tiene la responsabilidad social de formar ciudadanos y no sólo egresados profesionales. De acuerdo con Dewey (1954, p. 134), "las escuelas han de reconocer las necesidades de todas las clases de alumnos y dar a éstos una preparación que les haga llegar a ser ciudadanos prósperos y valiosos".

En los años setenta del siglo XX, Almond y Verba (1970, p. 469) observaron "tendencias cívicas 'aspirativas' en la cultura política mexicana". Lo que reportaron fue un cuadro inconsistente donde conviven el afecto general hacia el sistema político y la baja expectativa ciudadana respecto del trato igualitario por parte de las autoridades y de las realizaciones gubernamentales. Además de que los roles de súbdito y participante coexisten en ambivalencia entre los ciudadanos y la autoridad. El estudio, que se replicó en 1989, no aportó bases precisas para documentar cambios significativos en la cultura política mexicana (Craig y Cornelius, 1989, p. 342).

Al parecer, la ambivalencia es una característica en la cultura política del mexicano "aspirativo", pero alienado políticamente. Existe discrepancia entre los modelos "normativo" y "descriptivo" del sistema político en México (cfr. Craig y Cornelius, 1989, p. 376). No obstante, los estudios reportados posteriormente dieron cuenta de la presencia de una democracia constitucionalista en el país que privilegia la participación electoral, la presencia de partidos políticos y el voto, debido a que está condicionada a sus rendimientos económicos y sociales. Sin embargo, el ciudadano es pasivo, pues según la investigación realizada por Ai Camp (1999, p. 8), "los mexicanos esperan que la democracia les traiga justicia social y un mejor nivel de vida" y si las instituciones democráticas "fallan el cumplimiento de las expectativas de los ciudadanos, reforzarán sus creencias antidemocráticas". La conclusión fue que la ciudadanía sigue siendo alienada, aunque parcialmente participativa y aspirativa, tanto en el sentido democrático como social (García Clarck, 2001, p. 454).

El objetivo de la presente investigación fue analizar la cultura cívica de los alumnos de universidades interculturales, mediante su actitud ante situaciones emblemáticas, a fin de valorar formas de conducción en espacios comunes de convivencia entre universitarios. La pregunta de investigación fue: ¿los estudiantes de universidades interculturales tienen una perspectiva de cultura cívica incluyente, diversa y respetuosa ante circunstancias comunes a ellos y que favorecen la convivencia?

\section{Método}

El total de los participantes fue de 228 estudiantes de tres universidades interculturales: una situada en el sureste del país, otra en la región del Golfo de México y una más en la zona del centro. El rango promedio de edad fue de 20 y 22 años; $51 \%$ de los entrevistados fueron mujeres y $49 \%$ hombres.

\section{Procedimiento}

Se diseñó un cuestionario de ciudadanía que contiene 33 reactivos, en tres de sus componentes: cultura ciudadana, cultura cívica y cultura política, validado por cinco expertos en el tema. La primera parte es una escala Likert con cuatro opciones de respuesta: Sí, Tal vez, No y No lo sé, y mide el nivel de disposición para convivir con seis personas vulnerables en tres condiciones hipotéticas diferentes; contiene, además, 
un apartado para ordenar figuras de autoridad de acuerdo con el nivel de confianza que representa para los participantes. En la segunda, la cultura cívica incluyó seis casos donde plantearon dilemas en experiencias comunes que viven los universitarios. En la tercera se incorporó la cultura política. Se aplicaron los cuestionarios y se procedió al análisis en SPSS (Statistical Package for the Social Sciences). Los resultados obtenidos se registraron en el marco del proyecto PAPIIT N0401014-2 del IISUE-UNAM.

\section{Resultados}

Se calcularon las frecuencias y porcentajes de las situaciones dilema presentadas a los alumnos de las universidades interculturales. Los resultados se desagregan para cada caso, como sigue:

En la situación uno: "Repruebas una materia y un(a) profesor(a) te pide dinero a cambio de aprobarla, ¿qué haces?", la opción más cívica alcanzó $49.5 \%$ de las respuestas totales y casi la mitad restante optó por callarse, no hacer nada e incluso dar dinero al profesor. En la situación dos: "Repruebas una materia y un(a) profesor(a) te pide sexo a cambio de aprobarla, ¿qué haces?", la opción más cívica obtuvo las tres cuartas partes de las respuestas totales y poco más de la cuarta parte se ubicó en las que eran menos cívicas, resaltando en éstas la de acceder al chantaje (véase Tabla 1).

Tabla 1. Frecuencia y porcentajes para situaciones dilema 1 y 2.

\begin{tabular}{|c|c|c|c|}
\hline$N=228$ & Opciones de respuesta & Frecuencia & $\overline{\%}$ \\
\hline \multirow{5}{*}{$\begin{array}{l}\text { Situación } 1 . \\
\text { Repruebas una materia } \\
\text { y un(a) profesor(a) te } \\
\text { pide dinero a cambio de } \\
\text { aprobarla, ¿qué haces? }\end{array}$} & $\begin{array}{l}\text { Lo(a) denuncias a las autoridades por } \\
\text { corrupto(a). }\end{array}$ & 113 & 49.5 \\
\hline & No le das dinero y te quedas callado(a). & 43 & 19.0 \\
\hline & $\begin{array}{c}\text { Te sientes angustiado(a) y prefieres no } \\
\text { hacer nada. }\end{array}$ & 41 & 18.0 \\
\hline & Le das dinero al profesor(a) con tal de aprobar. & 31 & 13.5 \\
\hline & Total & 228 & 100.0 \\
\hline \multirow{5}{*}{$\begin{array}{l}\text { Situación } 2 \text {. } \\
\text { Repruebas una materia } \\
\text { y un(a) profesor(a) te } \\
\text { pide sexo a cambio de } \\
\text { aprobarla, ¿qué haces? }\end{array}$} & $\begin{array}{l}\text { Lo(a) denuncias a las autoridades por } \\
\text { corrupto(a). }\end{array}$ & 165 & 72.4 \\
\hline & No accedes y te quedas callado(a). & 29 & 12.7 \\
\hline & $\begin{array}{c}\text { Te sientes angustiado(a) y prefieres no } \\
\text { hacer nada. }\end{array}$ & 20 & 8.8 \\
\hline & $\begin{array}{l}\text { Accedes a tener sexo con el (la) profesor(a) con } \\
\text { tal de aprobar. }\end{array}$ & 14 & 6.1 \\
\hline & Total & 228 & 100.0 \\
\hline
\end{tabular}

Fuente: Elaboración propia. 
En la situación tres: "Estás presentando un examen para el que no estudiaste, en eso el (la) profesor(a) sale del salón, ¿entonces tú...?", la opción más cívica obtuvo casi las tres cuartas partes de las respuestas totales y poco más de la cuarta parte restante se inclinó por las opciones menos cívicas. En esta condición de dilema en la escuela parece más viable la conducta de sacar los apuntes que la de copiar, lo que es interesante es que son muy pocos los que entregarían el examen en blanco.

En la situación cuatro: “En tu trabajo actual tienes una fotocopiadora a tu disposición, entonces...", la respuesta más cívica alcanzó poco más de las tres cuartas partes y poco menos de la cuarta parte se distribuyó entre las menos cívicas, resaltando la relacionada con vender las fotocopias (Tabla 2 ).

Tabla 2. Frecuencia y porcentajes para situaciones dilema 3 y 4.

\begin{tabular}{|c|c|c|c|}
\hline$N=228$ & Opciones de respuesta & Frecuencia & $\%$ \\
\hline \multirow{5}{*}{$\begin{array}{c}\text { Situación } \mathbf{3} \text {. } \\
\text { Estás presentando un } \\
\text { examen para el que no } \\
\text { estudiaste, en eso el (la) } \\
\text { profesor(a) sale del salón, } \\
\text { ¿entonces tú...? }\end{array}$} & $\begin{array}{c}\text { Contestas el examen de acuerdo con lo } \\
\text { que sabes. }\end{array}$ & 156 & 68.4 \\
\hline & Lo entregas en blanco. & 9 & 4.0 \\
\hline & $\begin{array}{c}\text { Sacas tus apuntes y buscas las respuestas } \\
\text { del examen. }\end{array}$ & 37 & 16.2 \\
\hline & $\begin{array}{l}\text { Copias las respuestas del examen de } \\
\text { otro compañero. }\end{array}$ & 26 & 11.4 \\
\hline & Total & 228 & 1.00 .0 \\
\hline \multirow{5}{*}{$\begin{array}{c}\text { Situación } 4 . \\
\text { En tu trabajo actual } \\
\text { tienes una fotocopiadora } \\
\text { a tu disposición, entonces: }\end{array}$} & $\begin{array}{l}\text { Fotocopias sólo para cuestiones } \\
\text { laborales. }\end{array}$ & 167 & 73.2 \\
\hline & $\begin{array}{c}\text { Fotocopias documentos } \\
\text { personales. }\end{array}$ & 22 & 9.6 \\
\hline & Vendes las fotocopias. & 25 & 11.0 \\
\hline & Fotocopias para tus amigos. & 14 & 6.2 \\
\hline & Total & 228 & 100.0 \\
\hline
\end{tabular}

Fuente: Elaboración propia. 
En la situación cinco: "Eres funcionario público que puede contratar a un familiar tuyo muy cercano que está desempleado, ¿qué decides?", la opción más cívica obtuvo las tres cuartas partes de las respuestas totales y el 30\% restante se distribuyó en las menos, siendo más alta la de contratar aun cuando no se cumplen los requisitos (véase Tabla 3).

En la situación seis, "Si al pagar impuestos consideras que es mucho dinero, ¿qué haces?", hubo mayor variabilidad en las respuestas de los participantes; puede apreciarse que poco menos de la mitad respondió la opción más cívica y poco más del $50 \%$ se distribuyó entre las menos cívicas, resaltando dos alternativas: buscar un contador para pagar menos y no pagar el monto porque la persona cree que hay mal uso de los recursos.

Las opciones relativas a la mayor cultura cívica de los alumnos tuvieron variaciones de acuerdo con el caso planteado y el nivel de involucramiento de los participantes en cada uno de ellos.

Tabla 3. Frecuencia y porcentajes para situaciones dilema 5 y 6.

\begin{tabular}{|c|c|c|c|}
\hline$N=228$ & Opciones de respuesta & Frecuencia & $\%$ \\
\hline \multirow{5}{*}{$\begin{array}{l}\text { Situación } \mathbf{5} \text {. } \\
\text { Eres funcionario público } \\
\text { que puede contratar a un } \\
\text { familiar tuyo muy cercano } \\
\text { que está desempleado, } \\
\text { ¿qué decides? }\end{array}$} & $\begin{array}{l}\text { Lo recomiendas con algún conocido de otra } \\
\text { institución. }\end{array}$ & 159 & 70.0 \\
\hline & Le sugieres que busque trabajo en otro lugar. & 31 & 13.6 \\
\hline & Te das por enterado y no haces nada. & 5 & 2.0 \\
\hline & $\begin{array}{l}\text { Lo contratas inmediatamente aun cuando no } \\
\text { cumple con los requisitos. }\end{array}$ & 33 & 14.4 \\
\hline & Total & 228 & 100.0 \\
\hline \multirow{5}{*}{$\begin{array}{c}\text { Situación } \mathbf{6} \text {. } \\
\text { Si al pagar impuestos } \\
\text { consideras que es mucho } \\
\text { dinero, ¿qué haces? }\end{array}$} & Pagas el monto. & 106 & 46.6 \\
\hline & $\begin{array}{l}\text { Buscas un contador para pagar menos } \\
\text { impuestos. }\end{array}$ & 63 & 27.6 \\
\hline & $\begin{array}{l}\text { Evitas el pago de impuestos porque tienes } \\
\text { otras prioridades. }\end{array}$ & 22 & 9.6 \\
\hline & $\begin{array}{l}\text { No pagas el monto porque crees que hay mal } \\
\text { uso de recursos. }\end{array}$ & 37 & 16.2 \\
\hline & Total & 228 & 100.0 \\
\hline
\end{tabular}

Fuente: Elaboración propia. 
En la primera situación, reprobar una materia y que el profesor te pida dinero es una condición que involucra directamente al participante, al reprobar. Las respuestas presentaron variaciones, ya que casi la mitad de los alumnos estaría dispuesta a incurrir en una falta cívica. En el caso en que el profesor pide sexo para aprobar, la mayoría optó por la respuesta más cívica, pero poco más de la cuarta parte dijo estar dispuesto a cometer una falta e incluso aceptar con tal de aprobar. En la condición del pago de impuestos se reportó la mayor variabilidad de respuestas y hubo quienes afirmaron que buscarían un contador para pagar menos, o bien, no cubrir el monto porque hay mal uso de los recursos. En aquellas donde el participante se percibe menos involucrado, las respuestas cívicas obtuvieron el porcentaje más alto; destacan tres: presentar un examen, el uso de la fotocopiadora y ser funcionario público.

\section{A manera de cierre}

El propósito de la investigación fue identificar la actitud cívica de los alumnos de universidades interculturales en situaciones dilema, a partir de la cual se encontró coincidencia con el plano de la subjetividad de la que habla García Clarck (2001), ya que la cultura cívica de los alumnos muestra variaciones de acuerdo con el nivel de involucramiento que perciben en la sociedad: si les afecta directamente o no y dependiendo de ello orientan su valores y actitudes para la convivencia armónica. Así también con lo que reportaron Almond y Verba (1970) respecto de que los participantes ofrecieron respuestas variables y actitudes asimétricas que regulan su civismo.

Los hallazgos reafirman la presencia de ambivalencia como característica en la cultura política del mexicano "aspirativo" pero alienado políticamente, propias de las sociedades modernas (García Clarck, 2001, p. 454). La conducta cívica tiene componentes históricos, culturales y sociales de estudio para analizar la ciudadanía en términos de obligaciones y responsabilidades a fin de asegurar la convivencia armónica en universitarios interculturales.

\section{Referencias}

Almond, G. \& Verba, S. (1970). La cultura cívica. Estudio sobre la participación política democrática en cinco naciones. Madrid: Fundación de Estudios Sociales y de Sociología Aplicada.

Camp, R. A. (1999). La democracia vista a través de México. Este País, 100, 2-8.

Craig, A. \& Cornelius, W. (1989). Political culture in Mexico: continuities and revisionist interpretations. The civic culture revisited. California: Sage Publications.

Dewey, J. (1954). El niño y el programa escolar. Buenos Aires: Losada.

García Clarck, R. (2001). Bases para un diagnóstico de la cultura cívica en México. Sociológica, 16(4546), 445-456.

Marshall, T. (1950). Citizenship and Social Class and Other Essays. USA: Cambridge University Press.

UNAM-DGAPA. Proyecto PAPIIT N0401014-2, Ciudadanía en Educación Superior. Coordinador: Dr. Juan Manuel Piña Osorio del IISUE. 